\title{
Hybrid TIE/FDTD Method for Open Boundary Coupling Between Isolation Islands
}

\author{
Chun-Te Wu, Yi-Hsin Pang, and Ruey-Beei Wu, Senior Member, IEEE
}

\begin{abstract}
The two-dimensional finite-difference time-domain (2D-FDTD) method is extended to take into account the radiation effects due to the open boundary of PCBs on ground bounce. A time-domain equivalence principle for the structures with narrow apertures is proposed to divide the original ground bounce problem into the interior open-circuit problem and the exterior short circuit problem, which are handled by the 2D-FDTD method and the integral equation formulation, respectively. An efficient simulation procedure is established by casting equivalent inductances and capacitances with phase retardation into 2D-FDTD. Simulation results for the radiation effects due to open boundary or among isolated power/ground planes are presented and validated by measured data.
\end{abstract}

Index Terms-Finite-difference time-domain (FDTD), integral equation formulation, power islands, signal integrity.

\section{INTRODUCTION}

W ITH the increase in speed, performance, density, and package complexity, parasitic effects of the interconnections in printed circuit boards (PCBs) are becoming more critical. The National Technology Roadmap for Semiconductors (NTRS) projects predicts the advent of integrated chips with feature size of $50 \mathrm{~nm}$, supply voltage of $0.6 \mathrm{~V}$, and chip clock frequency of $10 \mathrm{GHz}$ by the year of 2012 [1]. In view of the high frequency and small noise budget due to the low power supply, the signal integrity issues resulted from parasitic effects have attracted great interest. Especially, the voltage variations between the power and ground planes due to the fast switching of the current flow, also known as delta-I noise, simultaneously switching noise, and ground bounce, increases significantly and must be well controlled in the very beginning of the design phase [2]-[5].

In common multilayer PCBs, there are many vias between two parallel plates to serve for the signal and/or power transmission in the vertical direction. Extensive studies have been presented in the literature and most of them focus on the ground bounce excited and coupled due to the presence of the vias. In light of the narrow separation between the parallel plates, an

Manuscript received March 21, 2003; revised January 15, 2004 and November 3, 2004. This work was supported in part by the Ministry of Education under Grant 89-E-Fa06-2 and in part by the National Science Council, R.O.C., under Grant NSC 89-2213 E002-197.

C.-T. $\mathrm{Wu}$ is with the Department of Communication Engineering, Da-Yeh University, Chang-Hua 515, Taiwan, R.O.C. (e-mail: samuel@mail.dyu.edu.tw).

Y.-H. Pang is with the Industrial Technology Research Institute, Hsinchu 310, Taiwan, R.O.C. (e-mail: yhpang@itri.org.tw).

R.-B. Wu is with the Department of Electrical Engineering and Graduate Institute of Communication Engineering, National Taiwan University, Taipei, 10617,Taiwan, R.O.C. (e-mail: rbwu@ew.ee.ntu.edu.tw).

Digital Object Identifier 10.1109/TADVP.2006.871171 efficient approach based on two-dimensional finite-difference time-domain (FDTD) method is possible for practical problems of thousands of vias [6].

In addition to vias, the presence of narrow slots is common to modern PCBs. For example, cutting a power plane into several areas provides multiple power distribution. Another is isolated power or ground plane areas, called voltage islands, to isolate a noisy or sensitive circuit from others [7]. However, signal lines have to cross the slots in order to communicate between different areas. The presence of slots significantly disturbs the return current path [8]. Simple equivalent circuit model was proposed to simulate the mode conversion mechanism between microstrip line mode and the slot line mode [9]. In multilayer structures, the signal crossing the slot will excite the parallel plate modes which exhibit as ground bounce between the power and ground planes. Mode conversion happens among microstrip mode, slotline mode, and the parallel plate mode. Recently, an efficient model based on equivalent circuits and two-dimensional FDTD analysis has been successfully established to simulate this complicated mode conversion mechanism [10].

In reality, the power and ground planes in PCBs must be finite in size. The edges of the finite-size plate will not only cause signal reflection but also radiation. It can serve as electromagnetic interference (EMI) source as well as receptor. For structures with isolated power islands, the coupling among the edges may become a major source of noise and should be taken into account. As a result, mode coupling and conversion in terms of parallel plate mode will happen among discontinuities of vias, slots in split plane, and edges of the finite-size plates [11].

The complicated mode conversion mechanism has been investigated using the three-dimensional FDTD method [11]-[13]. However, the packaging structures usually include elements of drastically different scales, say, the microstrip and slotline are usually very narrow as compared with the parallel plate. The FDTD method is usually incapable of accurately modeling the structure details and if so, will need long computation time and large computer memory. On the other hand, structures of different scales can be much powerfully modeled by integral-equation based methods, like mixed potential integral equation (MPIE) [14] and partial element equivalent circuit (PEEC) [15], [16]. However, modeling of the finite-size plates in PEEC calls for a great number of unknowns associated with a full matrix, which will easily exhaust the available computer resources. Both methods, although flexible and general, still find difficulty in dealing with practical packaging structures.

In a PCB, the electric field between ground/power planes is uniform in the longitudinal direction except near the edge 


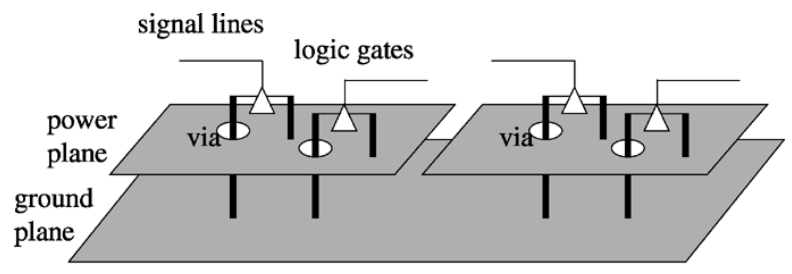

(a)

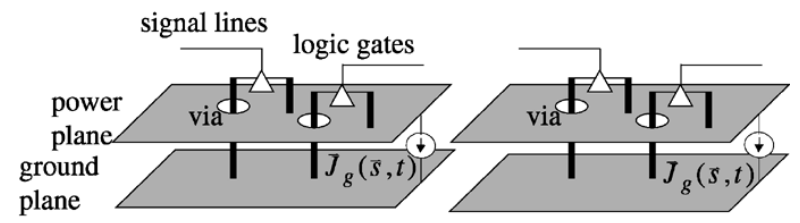

(b)

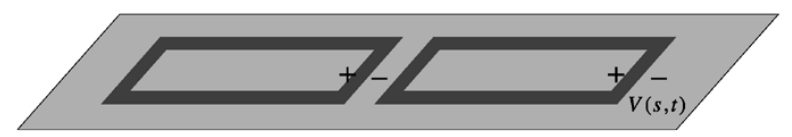

(c)

Fig. 1.Typical PCB structures with separated poser island. (a) Original problem. (b Interior open-circuit problem. (c) Exterior-short circuit problem.

due to the fringing effects. A more efficient approach should be based on two-dimensional FDTD [6]. However, such an approach usually puts perfect magnetic conductor (PMC) on the edge and neglects the radiation effects. This paper will propose a new efficient analysis scheme. It hybridizes the two-dimensional FDTD method for the ground bounce propagation between parallel plates and the time-domain equivalent circuits for electromagnetic wave radiation/coupling due to the edges of the finite plates. The FDTD and edge opening simulations can be performed separately and linked together at each computation time step. The effects of the open edges along the boundary on signal propagation are thus characterized.

\section{HYBRID TIE/FDTD METHOD}

\section{A. Equivalent Problems}

Fig. 1(a) shows a typical PCB structure with separated power planes of finite size. The ground plane is much larger in size and assumed to be infinite in this paper. Due to the wave leakage from the aperture opening along the boundary, there will be some coupling between the two power islands. It is desired to derive an efficient formulation directly applicable for the time domain analysis.

The problem can be decomposed into an interior open circuit problem and an exterior short circuit problem shown in Fig. 1(b) and (c), respectively. Let $\vec{s}$ denote the position vector on the boundary surface. In the interior problem, the parallel plates are assumed to be ideally open at the boundary but with the impressed surface current density $\vec{J}_{g}(\vec{s}, t)=-\hat{n} \times \vec{H}(\vec{s}, t)$, where $\hat{n}$ is the unit outwards normal vector and $\vec{H}(\vec{s}, t)$ is the magnetic field intensity on the boundary surface.

In the exterior problem, the aperture is assumed to be short circuited but with an equivalent voltage source $V(\vec{s}, t)$. The voltage is directly related to the electric field on the plate boundary by $V(\vec{s}, t)=-d \cdot E_{z}(\vec{s}, t)$. Let the resultant magnetic field intensity along the boundary be $\vec{H}^{s}(\vec{s}, t)$. The surface current density flowing across the shorted aperture can be given by $\vec{J}(\vec{s}, t)=\hat{n} \times \vec{H}^{s}(\vec{s}, t)$, as shown in Fig. 1(c). According to the field continuity between $\vec{H}(\vec{s}, t)$ and $\vec{H}^{s}(\vec{s}, t)$, the surface currents in the interior and exterior problems require $\vec{J}_{g}(\vec{s}, t)+\vec{J}(\vec{s}, t)=\overrightarrow{0}$. In other words, the total current flow across the aperture is zero, which justifies the equivalence between the original and the equivalent problems.

\section{B. Interior Open-Circuit Problem}

Consider the problem in Fig. 1(b). In common cases that $d$ is much smaller than the wavelength and the size of the plate, it is reasonable to assume that the electric field between the parallel plates contains $E_{z}(x, y, t)$ component only. In reality, the ground bounce between the parallel plates is measured by

$$
V(x, y, t)=-d \cdot E_{z}(x, y, t) .
$$

The surface current density on the upper plane $\vec{J}(x, y, t)$ is related to the magnetic field intensity between the two plates by

$$
\vec{J}(x, y, t)=-\hat{z} \times \vec{H}(x, y, t) .
$$

Note that the current density on the lower plane is $-\vec{J}(x, y, t)$.

From the Maxwell equation between the electric and magnetic fields, it is not difficult to derive the relation between the voltage and surface current density. Without loss of generality, assume the plate is rectangular and divided into $N_{x} \cdot N_{y}$ small cells of size $\Delta_{x} \times \Delta_{y}$. The relation can be more physically written in terms of the equivalent $L C$ network [17] as shown in Fig. 2. The capacitance and inductance in the network are given by

$$
C_{\mathrm{FDTD}}=\frac{\varepsilon \Delta_{x} \Delta_{y}}{d} \quad L_{\mathrm{FDTD}}^{x}=\frac{\mu d \Delta_{x}}{\Delta_{y}} \quad L_{\mathrm{FDTD}}^{y}=\frac{\mu d \Delta_{y}}{\Delta_{x}} .
$$

For the nodal voltages along the boundary, the impressed current source should be accounted for to model the electromagnetic coupling and/or radiation from the open edge. They satisfy the following form:

$$
\kappa C_{\mathrm{FDTD}} \frac{d}{d t} V(\vec{s}, t)=I_{\mathrm{FDTD}}^{\mathrm{inc}}(t)-I_{g}(\vec{s}, t) .
$$

Here, $\kappa$ is the area fill factor. The value is $\kappa=1 / 2$ for the node along the "straight" part of the boundary, while $\kappa=1 / 4$ for the node at the corner. The term $I_{\mathrm{FDTD}}^{\mathrm{inc}}(t)$ denotes sum of the current in the interior problem injected to the node. Say, for the node $\vec{s}=\left(x_{M}, y_{j}\right)$ on the right side of the boundary shown in Fig. 2

$$
\begin{aligned}
I_{\mathrm{FDTD}}^{\mathrm{inc}}(t)=I^{x}\left(x_{N_{x}-\frac{1}{2}}, y_{j}, t\right)+ & I^{y}\left(x_{N_{x}}, y_{j-\frac{1}{2}}, t\right) \\
& -I^{y}\left(x_{N_{x}}, y_{j+\frac{1}{2}}, t\right) .
\end{aligned}
$$

The impressed current $I_{g}(\vec{s}, t)=-\int \vec{J}_{g}(\vec{s}, t) \cdot \hat{z} d \vec{s}$ is available from the exterior problem and should be accounted for when updating the voltage at the boundary. 


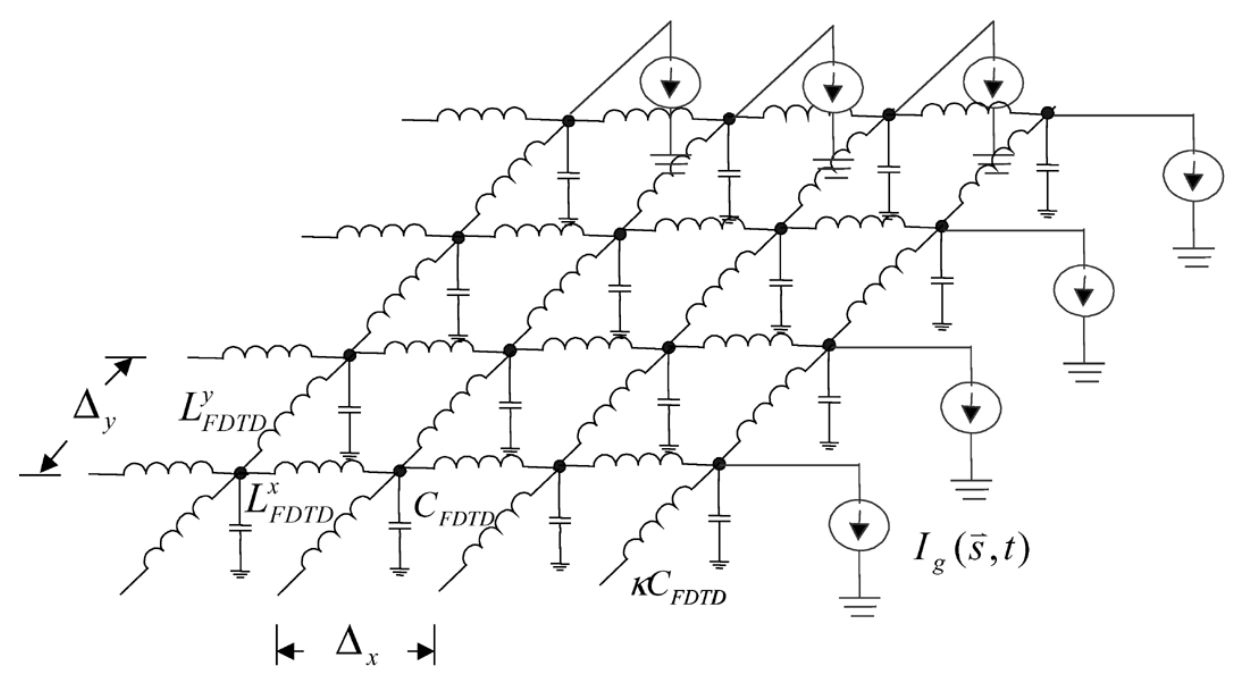

Fig. 2. Equivalent $L C$ network for the interior problem.

\section{Exterior Short-Circuit Problem}

The voltage across the aperture along the plate boundary can be treated as an equivalent magnetic current density

$$
\vec{M}=\vec{E} \times \hat{n}=-\frac{V(\vec{s}, t)}{d} \hat{s}
$$

where $\hat{s}$ denotes the unit vector tangential to the boundary counter-clockwise. In common cases, the separation $d$ between the two plates is very small and can be neglected. It is a good approximation to model the problem as the excited field by the magnetic current source on a planar ground plane. If the ground plane is large enough to apply image theory, the ground plane can be replaced by doubling the magnetic current, which is illustrated in Fig. 3.

To facilitate the derivation, it is easier to employ the time-harmonic analysis. All the variables in the following two sections are expressed in terms of the phasor notation. The scattered magnetic field on the aperture due to the magnetic current can be expressed as [18]

$$
\vec{H}^{s}=\frac{\nabla \nabla \cdot \vec{F}+k^{2} \vec{F}}{j \omega \mu}=-\nabla \Psi-j \omega \varepsilon \vec{F}
$$

where the magnetic vector potential

$$
\vec{F}(\vec{s})=\int_{\Gamma} G\left(\left|\vec{s}-\vec{s}^{\prime}\right|\right) \vec{M}\left(\vec{s}^{\prime}\right) d \vec{s}^{\prime}
$$

in which $G(R)=e^{-j k R} / 2 \pi R$ is the free space Green's function in the presence of the ground plane, $k$ is the free space wave number, and $\Gamma$ denotes the boundary surface.

In (7), the magnetic scalar potential $\Psi$ is introduced as the dual of electric potential. After some algebraic simplification, it can be expressed as

$$
\Psi(\vec{s})=\frac{1}{\mu} \int_{\Gamma} G\left(\left|\vec{s}-\vec{s}^{\prime}\right|\right) \rho_{m}\left(\vec{s}^{\prime}\right) d \vec{s}^{\prime}
$$

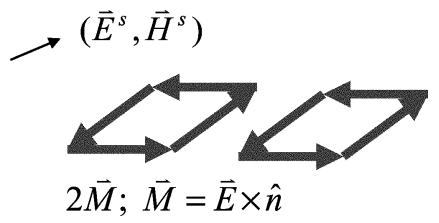

Fig. 3. Equivalent magnetic current source and resultant electromagnetic field for exterior problem.

where $\rho_{m}$ is the so-called surface magnetic charge density and is defined by

$$
\rho_{m}(\vec{s})=-\frac{1}{j \omega} \nabla \cdot \vec{M}(\vec{s}) .
$$

The derivation to (9) is possible since $G$ is a function of $\left|\vec{s}-\vec{s}^{\prime}\right|$ only and the vector identity

$$
\begin{aligned}
\int_{\Gamma}(\nabla \cdot G) \vec{M} d \vec{s}^{\prime} & =\int_{\Gamma}\left(-\nabla^{\prime} \cdot G\right) \vec{M} d \vec{s}^{\prime} \\
& =-\int_{\Gamma} \nabla^{\prime} \cdot(G \vec{M}) d \vec{s}^{\prime}+\int_{\Gamma} G \nabla^{\prime} \cdot \vec{M} d \vec{s}^{\prime}
\end{aligned}
$$

where $\nabla^{\prime}$ means the del operator acting on the primed coordinate $\vec{s}^{\prime}$. It is obvious that the first term in the right-hand side of (11) is zero since either $\Gamma$ is a closed surface or $\vec{M}=0$ at the end.

The surface current density across the aperture from the exterior problem will be $\hat{n} \times \vec{H}^{s}(\vec{s})$. Since the total current flows across the aperture should be zero, the integral equation governing the impressed surface current density $\vec{J}_{g}(\vec{s})$ in the interior problem and the voltage source $V(\vec{s})$ in the exterior problem is given by

$$
\vec{J}_{g}(\vec{s})+\hat{n} \times \vec{H}^{s}(\vec{s})=0 .
$$

Note that $\vec{H}^{s}(\vec{s})$ is related to $V(\vec{s})$ by (7)-(10). 


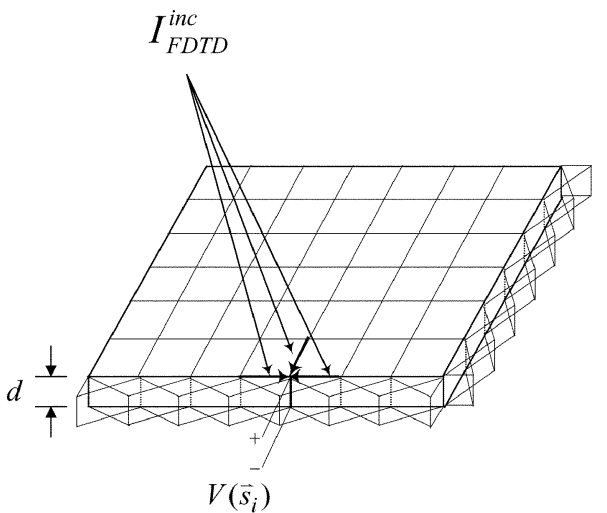

(a)

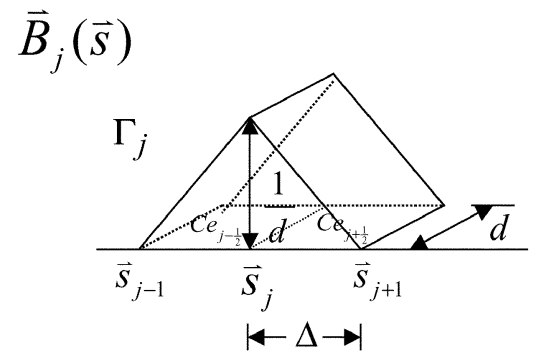

(b)

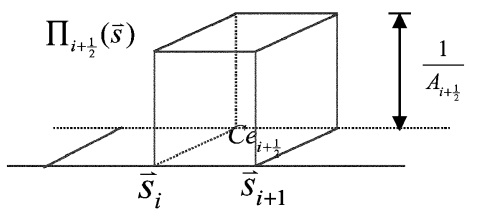

(c)

Fig. 4. (a) Grid meshing for the structure and node voltage $V\left(\vec{s}_{j}\right)$. (b) Roof-top basis for magnetic current. (c) Pulse basis for magnetic flux across the aperture.

\section{Moments Method Solution}

Assume that the magnetic currents is expressed as

$$
\vec{M}(\vec{s})=\sum_{j} V\left(\vec{s}_{j}\right) \vec{B}_{j}(\vec{s})
$$

where $\vec{B}_{j}(\vec{s})$ is the roof-top basis on the area of cells $C e_{j-(1 / 2)}$ and $C e_{j+(1 / 2)}$ as illustrated in Fig. 4. The surface magnetic charge density by (10) becomes

$$
\rho_{m}(\vec{s})=\sum_{j} \lambda\left(\vec{s}_{j+\frac{1}{2}}\right) \Pi_{j+\frac{1}{2}}(\vec{s}) .
$$

Here, $\Pi_{j+(1 / 2)}(\vec{s})$ is a pulse basis, being $1 /\left(d \cdot \mid \overrightarrow{s_{j+1}}-\vec{s}_{j}\right)$ on the cell $C e_{j+(1 / 2)}$ and zero elsewhere. $\lambda(\vec{s})$ denotes the magnetic flux over this cell and is related to the voltage by

$$
j \omega \lambda\left(\vec{s}_{j+\frac{1}{2}}, t\right)=V_{g}\left(\vec{s}_{j}\right)-V_{g}\left(\vec{s}_{j+1}\right) .
$$

Choosing the same basis functions (13) as the weighting function, the integral equation (12) yields to

$$
I_{g}\left(\vec{s}_{i}\right) \cong \int_{\Gamma_{i}} \vec{J}_{g}(\vec{s}) \cdot \hat{n} \times \vec{B}_{i}(\vec{s}) d \vec{s}=-\int_{\Gamma_{i}} \vec{H}^{s}(\vec{s}) \cdot \vec{B}_{i}(\vec{s}) d \vec{s}
$$

The right-hand side in (16) contains the contributions from two terms. For the contribution of magnetic scalar potential, it is not difficult to show that

$$
\begin{aligned}
& -\int_{\Gamma_{i}} \vec{B}_{i} \cdot \nabla \Psi d \vec{s} \\
& =-\int_{\Gamma_{i}} \nabla \cdot\left(\Psi \vec{B}_{i}\right) d \vec{s}+\int_{\Gamma_{i}} \Psi \nabla \cdot \vec{B}_{i} d \vec{s}=\int_{\Gamma_{i}} \Psi \nabla \cdot \vec{B}_{i} d \vec{s} \\
& =\frac{1}{\mu} \sum_{j} \lambda\left(\vec{s}_{j+\frac{1}{2}}\right)\left\{\int_{C e_{i-\frac{1}{2}}} \Pi_{i-\frac{1}{2}}(\vec{s})-\int_{C e_{i+\frac{1}{2}}} \Pi_{i+\frac{1}{2}}(\vec{s})\right\} \\
& \quad \times \int_{C e_{j+\frac{1}{2}}} G\left(\left|\vec{s}-\vec{s}^{\prime}\right|\right) \Pi_{j+\frac{1}{2}}\left(\vec{s}^{\prime}\right) d \vec{s}^{\prime} d \vec{s} .
\end{aligned}
$$

As a result, (16) becomes

$$
\begin{aligned}
I_{g}\left(\overrightarrow{s_{i}}\right) \cong \sum_{j}\left(L_{i+\frac{1}{2}, j+\frac{1}{2}}^{-1}-L_{i-\frac{1}{2}, j+\frac{1}{2}}^{-1}\right) \lambda\left(\vec{s}_{j+\frac{1}{2}}\right) & \\
& +j \omega \sum_{j} C_{i, j} V\left(\vec{s}_{j}\right)
\end{aligned}
$$

in which

$$
\begin{aligned}
L_{i+\frac{1}{2}, j+\frac{1}{2}}^{-1} & \equiv \frac{1}{\mu} \int_{C e_{i+\frac{1}{2}}} \int_{e_{j+\frac{1}{2}}} \frac{e^{-j k R}}{2 \pi R} \Pi_{i+\frac{1}{2}}(\vec{s}) \Pi_{j+\frac{1}{2}}\left(\vec{s}^{\prime}\right) d \vec{s} d \vec{s}^{\prime} \\
C_{i, j} & \equiv \varepsilon \int_{\Gamma_{i}} \int_{\Gamma_{j}} \frac{e^{-j k R}}{2 \pi R} \vec{B}_{i}(\vec{s}) \cdot \vec{B}_{j}\left(\vec{s}^{\prime}\right) d \vec{s} d \vec{s}^{\prime}
\end{aligned}
$$

\section{E. Time-Domain Integral Equation}

Physically, $C_{i, j}$ denotes the capacitive coupling between branch voltages $V\left(\vec{s}_{j}\right)$ and $V\left(\vec{s}_{i}\right), C_{i, i}$ is the self-capacitance, $L_{i+(1 / 2), j+(1 / 2)}$ denotes the mutual inductance between cells $C e_{i+(1 / 2)}$, and $C e_{j+(1 / 2)}$, and $L_{i+(1 / 2), i+(1 / 2)}$ is the self inductance of the cell $C e_{i+(1 / 2)}$. Due to the phase retardation term $e^{-j k R}=e^{-j \omega R / c}$, the effect of $C_{i, j}$ in the time domain happens at $(1 / c) R_{i, j}=(1 / c)\left|\vec{s}_{i}-\vec{s}_{j}\right|$ earlier, and similarly for $L_{i+(1 / 2), j+(1 / 2)}^{-1}$. Hence, from (18), the time-domain relation between $I_{g}\left(\vec{s}_{i}, t\right)$ and $V\left(\vec{s}_{i}, t\right)$ is given by

$$
\begin{aligned}
I_{g}\left(\overrightarrow{s_{i}}, t\right) \cong & \sum_{j} L_{i+\frac{1}{2}, j+\frac{1}{2}}^{-1} \lambda\left(\vec{s}_{j+\frac{1}{2}}, t-\frac{1}{c} R_{i+\frac{1}{2}, j+\frac{1}{2}}\right) \\
& -\sum_{j} L_{i-\frac{1}{2}, j+\frac{1}{2}}^{-1} \lambda\left(\vec{s}_{j+\frac{1}{2}}, t-\frac{1}{c} R_{i-\frac{1}{2}, j+\frac{1}{2}}\right) \\
& +\sum_{j} C_{i, j} \frac{d}{d t} V\left(\vec{s}_{j}, t-\frac{1}{c} R_{i, j}\right)
\end{aligned}
$$

where $C_{i, j}$ and $L_{i+(1 / 2), j+(1 / 2)}^{-1}$ are the dc-values obtained from (19) with $k=0$. By (15), the magnetic flux $\lambda\left(\vec{s}_{j+(1 / 2)}, t\right)$ satisfies

$$
\frac{d}{d t} \lambda\left(\vec{s}_{j+\frac{1}{2}}, t\right)=V\left(\vec{s}_{j}, t\right)-V\left(\vec{s}_{j+1}, t\right) .
$$




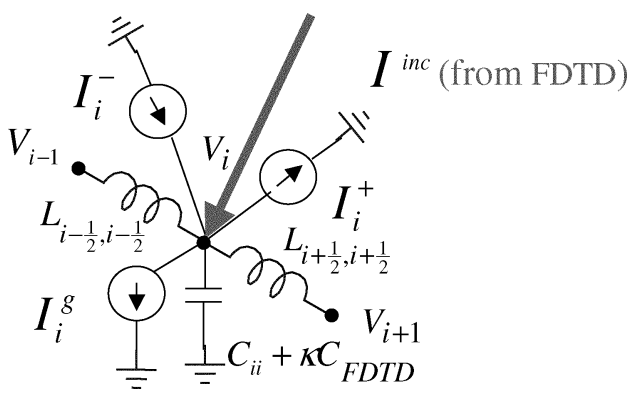

Fig. 5. Equivalent circuit for updating the node voltage at boundary.

By substituting (20) into (4), the equivalent circuit for the nodes along the boundary can be obtained and illustrated in Fig. 5. The desired updating equation is

$$
\begin{aligned}
\left(\kappa C_{\mathrm{FDTD}}+C_{i, i}\right) \frac{d V\left(\vec{s}_{i}, t\right)}{d t}= & I_{\mathrm{FDTD}}^{\mathrm{inc}}-L_{i+\frac{1}{2}, i+\frac{1}{2}}^{-1} \lambda\left(\vec{s}_{i+\frac{1}{2}}, t\right) \\
& +L_{i-\frac{1}{2}, i-\frac{1}{2}}^{-1} \lambda\left(\vec{s}_{i-\frac{1}{2}}, t\right) \\
& +\left[-I_{i}^{+}+I_{i}^{-}-I_{i}^{g}\right]
\end{aligned}
$$

where the terms in the bracket consist of the earlier contributions and are given by

$$
\begin{aligned}
I_{i}^{+} & =\sum_{j \neq i} L_{i+\frac{1}{2}, j+\frac{1}{2}}^{-1} \lambda\left(\vec{s}_{j+\frac{1}{2}}, t-\frac{1}{c}\left|\vec{s}_{i+\frac{1}{2}}-\vec{s}_{j+\frac{1}{2}}\right|\right) \\
I_{i}^{-} & =\sum_{j \neq i} L_{i-\frac{1}{2}, j-\frac{1}{2}}^{-1} \lambda\left(\vec{s}_{j-\frac{1}{2}}, t-\frac{1}{c}\left|\vec{s}_{i-\frac{1}{2}}-\vec{s}_{j-\frac{1}{2}}\right|\right) \\
I_{i}^{g} & =\sum_{j \neq i} C_{i, j} \frac{d}{d t} V\left(\vec{s}_{j}, t-\frac{1}{c}\left|\overrightarrow{s_{j}}-\overrightarrow{s_{i}}\right|\right) .
\end{aligned}
$$

\section{F. Time Marching Scheme}

The time marching scheme can be described as follows.

Step 1) Start from the voltage $V^{n-1}\left(\overrightarrow{s_{i}}\right)$ the magnetic flux $\lambda^{n-(1 / 2)}\left(\overrightarrow{s_{i+(1 / 2)}}\right)$ for all $i$ and the voltage $V_{\mathrm{FDTD}}^{n-1}$ and current $I_{\mathrm{FDTD}}^{n-(1 / 2)}$ in the interior problem. Here, the superscript denotes the time step.

Step 2) Update the voltage $V_{\mathrm{FDTD}}^{n}$ in the interior problem from $V_{\mathrm{FDTD}}^{n-1}$ and $I_{\mathrm{FDTD}}^{n-(1 / 2)}$ by employing Ampere's Law. Note that the voltage $V^{n-1}\left(\overrightarrow{s_{i}}\right)$ along boundary has yet to be determined since the Ampere's law is not applicable there.

Step 3) Update the voltage $V^{n}\left(\overrightarrow{s_{i}}\right)$ by (22).

Step 4) Update the magnetic flux $\lambda^{n+(1 / 2)}\left(\overrightarrow{s_{i+(1 / 2)}}\right)$ from $\lambda^{n-(1 / 2)}\left(\overrightarrow{s_{i+(1 / 2)}}\right), V^{n}\left(\overrightarrow{s_{i}}\right)$ by $(22)$.

Step 5) Update currents $I_{\mathrm{FDTD}}^{n+(1 / 2)}$ from $V_{\mathrm{FDTD}}^{n}$, and current $I_{\mathrm{FDTD}}^{n-(1 / 2)}$ by employing Faraday's Law.

It is worthy mentioning that Step 2) and 5) in the updating equations for voltage and current inside the FDTD unit cell are exactly the same as those in the conventional 2D-FDTD.

\section{NUMERICAL EXAMPLES}

\section{A. Comparison With Frequency Domain Full-Wave Solver}

A patch antenna with finite ground plane is used to test the present methodology. As shown in Fig. 6, the size of patch is

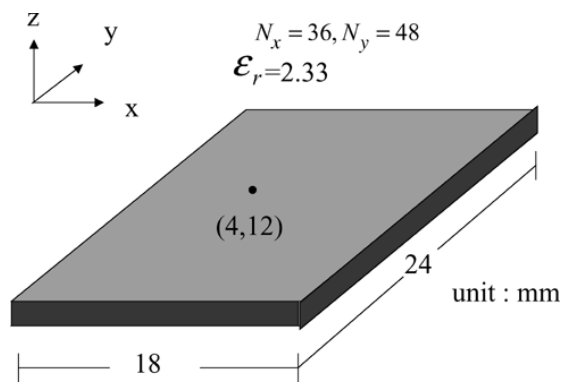

Fig. 6. Patch antenna of infinite ground plane used for simulation.

$1.8 \times 2.4 \mathrm{~cm}$, with the dielectric constant $\varepsilon_{r}=2.33$, and substrate thickness $d=1 \mathrm{~mm}$. In numerical simulation, the grid size of FDTD is $\Delta=0.5 \mathrm{~mm}$. The total simulation region is divided into $36 \times 48$ cells along $x$ and $y$ directions, respectively. The signal voltage source is a Gaussian pulse generator

$$
V_{s}(t)=\exp \left[-\left(\frac{t-t_{0}}{\sigma}\right)^{2}\right]
$$

in series with an internal resistance of $50 \Omega$ and is incident at $\vec{r}_{0}=(4 \mathrm{~mm}, 12 \mathrm{~mm})$. The parameters of Gaussian pulse are $\Delta t=1 \mathrm{ps}, t_{0}=50 \Delta t, \sigma=10 \Delta t$.

From the simulated waveform at $\vec{r}_{0}$, the input impedance to the patch structure $Z_{i}(\omega)$ is calculated by

$$
Z_{i}(\omega, z)=\frac{F\left[V\left(\vec{r}_{0}, t\right)\right]}{F\left[I\left(\vec{r}_{0}, t\right)\right]} .
$$

Hence, $S_{11}$ can be calculated by

$$
S_{11}(\omega)=\frac{Z_{i}(\omega)-50}{Z_{i}(\omega)+50} .
$$

Fig. 7 compares the results simulated by the present method and IE3D. ${ }^{1}$ The first null occurs at $5.3 \mathrm{GHz}$ for the present method and $5.22 \mathrm{GHz}$ for IE3D. It is found that the results by both methods are in good agreement. However, the null frequency of the present method is slightly higher than that predicted by IE3D, since the present method assumes that the magnetic current along the open boundary radiates in free space. The real relative permittivity $\varepsilon_{r}$ should be higher than one due to the presence of substrate.

\section{B. Experimental Validation}

A four-layer PCB of two isolation power islands is fabricated to investigate the coupled noise between islands. As shown in Fig. 8, the two power islands are of size $2.5 \times 3 \mathrm{~cm}$ and separated by $1 \mathrm{~mm}$, while the substrate is of thickness $d=1.2 \mathrm{~mm}$, and dielectric constant $\varepsilon_{r}=4.2$. The whole structure is symmetric.

In the experiment, a ramped step pulse of about 100-ps rise time is used as the excitation on port 1 . The time-domain reflectometry (TDR) and time-domain transmission (TDT) signals at ports 1 and 2, respectively, are measured by Tektronix 11801C. Since there are neither vias nor transmission lines between these two power islands, the measured signal at port 2, if any, could be contributed to the coupling of the open boundary.

\footnotetext{
${ }^{1}$ Zeland Software, Inc. Fremeont, CA.
} 


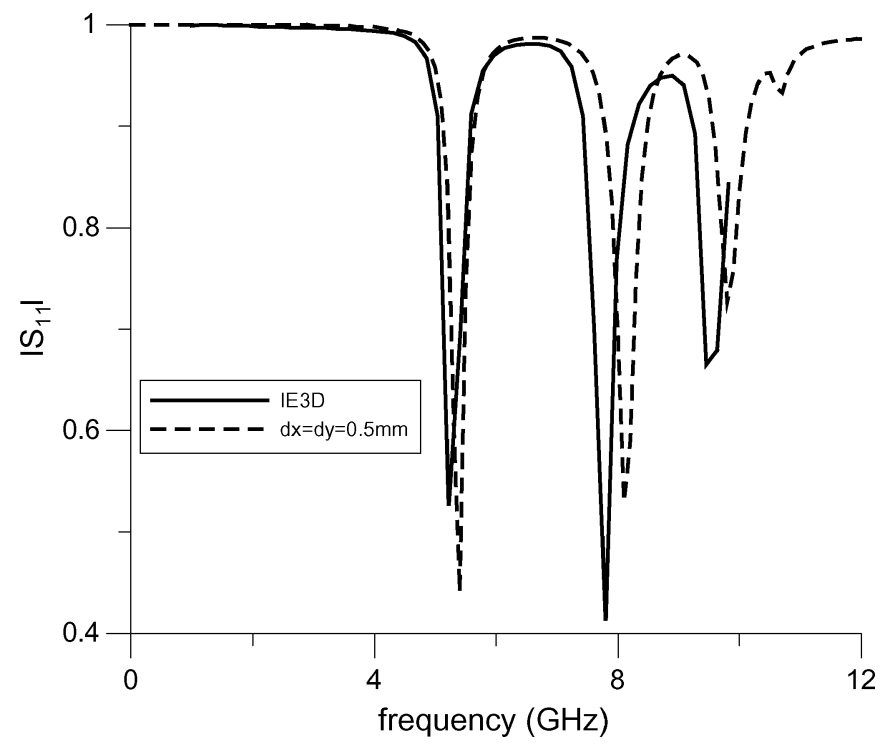

Fig. 7. Simulated results for $S_{11}$ by the present method and a frequency-domain full-wave solver IE3D.

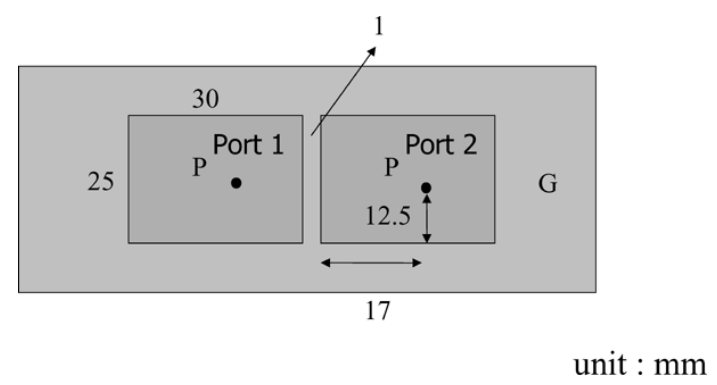

(a)

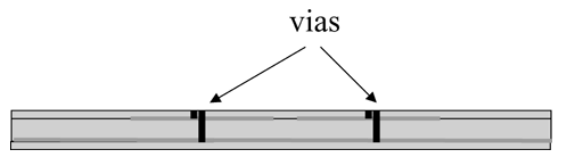

(b)

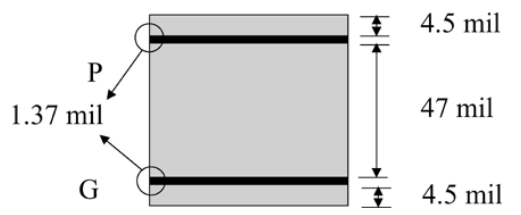

(c)

Fig. 8. Test PCB of isolation power plane used for experiment and simulation. (a) Top view. (b) Side view. (c) Thickness of the substrate and metal plane.

In numerical simulation, the measured input waveform is used as the excitation signal. The total simulation region is divided into $61 \times 25$ cells with grid size $\Delta=1 \mathrm{~mm}$ along $x$ and $y$ directions, respectively. Noting that the power plane for this structure is embedded into the dielectric, the relative permittivity for the exterior problem is chosen as $\varepsilon_{r}=4.2$. A better value should be somewhat lower than 4.2 , since the material is not distributed in the whole space. The simulation is performed to 8000 time steps with $\Delta t=1$ ps to get a frequency resolution of $0.125 \mathrm{GHz}$.

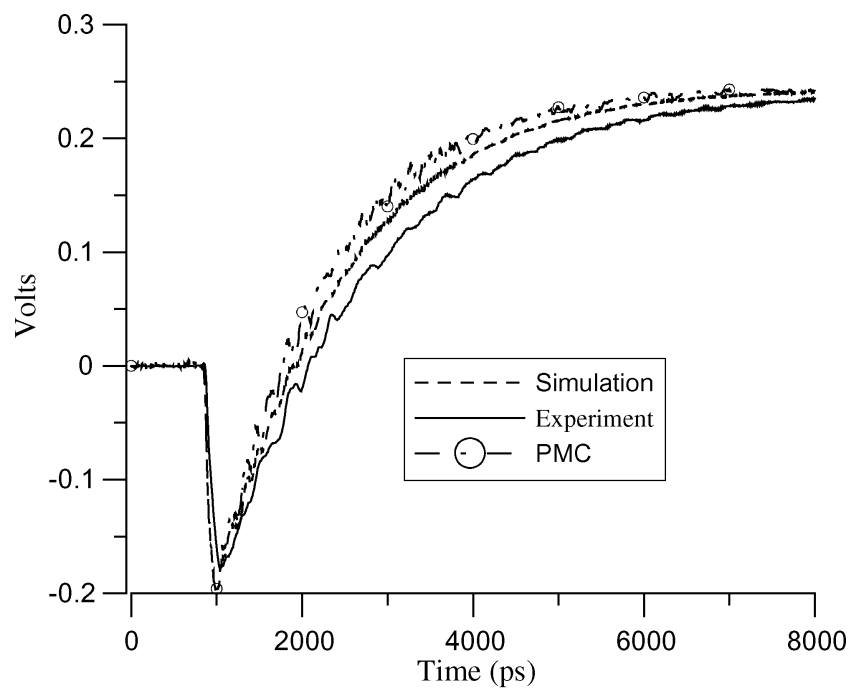

(a)

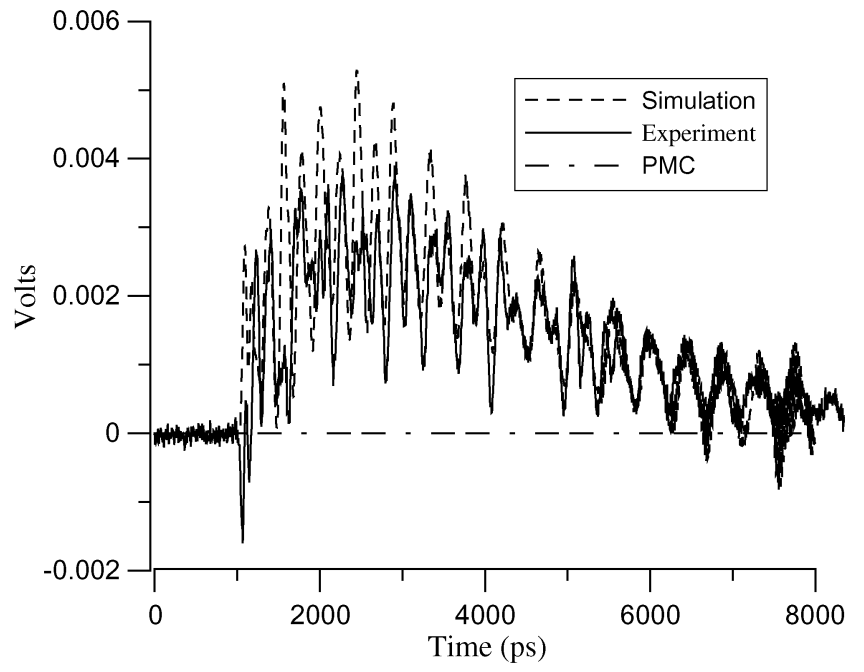

(b)

Fig. 9. Measured and simulated waveforms of (a) TDR signal at port 1 and (b) TDT signal at port 2 .

Fig. 9(a) and (b) shows the results at TDR and TDT ports, respectively. These curves denote the results obtained by simulation with radiation and PMC boundary, and experiment. They exhibit different $R C$ time constants at the TDR port. The value is close to 1162 ps for the case of PMC boundary. This can be justified since the capacitance is calculated to be $C=\varepsilon(A / d) \approx$ $23.24 \mathrm{pF}$ by parallel-plate formula, and the resistance $R=50 \Omega$. The $R C$ time constant will be larger in the present method, since the fringing effect enlarges the effective area of patch. It is noted from the figure that the $R C$ time constant of experiment is even longer, which might be contributed to some additional loss of measurement system and PCB conductor and dielectric.

At the TDT port, the conventional method, which assumes PMC boundary fails to predict any coupled noise. In the present case that the step voltage of the signal is $0.25 \mathrm{~V}$, the coupling noise level at port 2 is measured to be $4 \mathrm{mV}$ as opposed to $5.3 \mathrm{mV}$ by the present simulation method. In other words, the generated coupled noise between isolation islands for the present case may achieve about $2 \%$ of the input level. The 


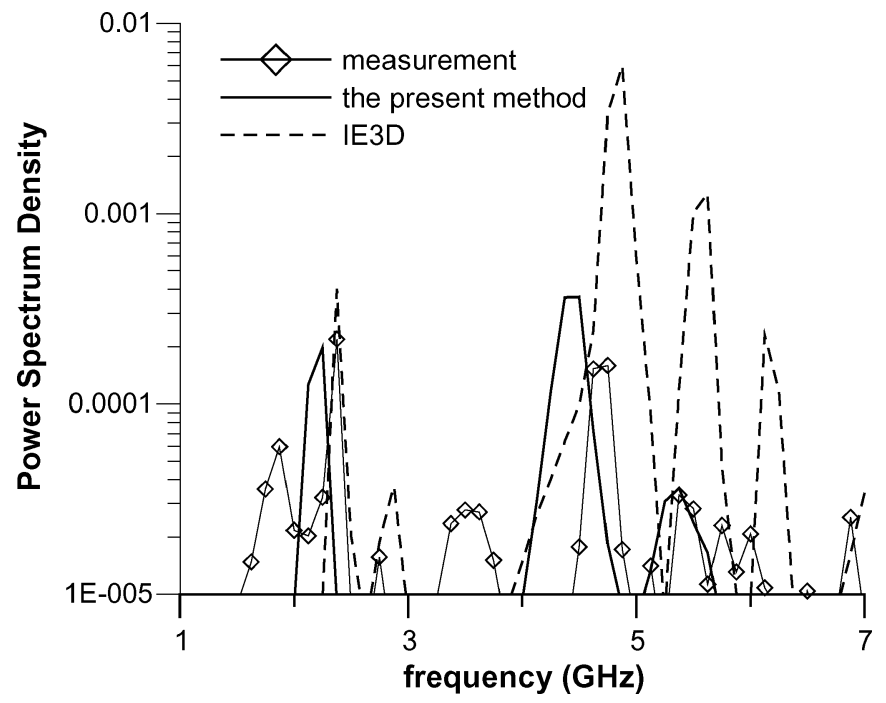

Fig. 10. Simulated measured power spectrum density. Comparisons among measurement, the present method, and IE3D.

over-estimation in the simulation result can be contributed to the negligence of the loss by the substrate, conductor, and radiation.

Fig. 10 shows the power spectrum density of the TDT signal obtained by the measurement and the present method. Also shown for comparison are the results by frequency-domain full-wave solver IE3D, for which the power spectrum density is calculated by the multiplication of $S_{21}$ and power spectrum density of input waveform. Both the present simulation and IE3D are executed on the same computer with a CPU of Pentium III at $1 \mathrm{GHz}$. The present method needs $450 \mathrm{~s}$ to calculate the response. Based on the same gridding and the same frequency resolution, IE3D needs $33062 \mathrm{~s}$ to complete the simulation, almost 74 times longer. In addition, it can be seen from the figure that the results predicted by IE3D deviate from the measured data much more significantly than those by the present simulation method.

There are some peaks to signify the various resonance modes in Fig. 10. Since the position of excitation is located at the symmetric line of the short edge, the first two peaks correspond to $\mathrm{TM}_{10}$ and $\mathrm{TM}_{20}$ modes and should occur at 2.375 and $4.714 \mathrm{GHz}$, respectively. The agreement between measurement and simulation demonstrates the accuracy of the present method. The frequency of the peak coupling noise is a little bit lower than the measurement, which may be partly contributed to using $\varepsilon_{r}=4.2$ for the exterior problem.

\section{CONCLUSION}

A new methodology is presented to simulate the signal integrity issues between isolation islands due to radiation coupling from the power plane boundary. The methodology can be cast into two-dimensional FDTD to analyze the radiation problems of PCB. Favorable agreement between numerical and experimental results has demonstrated the accuracy of this method.
Although the coupled noise caused by radiation is less than $2 \%$, the effects may severely deteriorate the signal integrity of other islands at some resonant frequencies.

\section{REFERENCES}

[1] Semiconductor Industry Association. (1997). [Online] Available: http://www.macs.ee.mcgill.ca/ roberts/PUBLIC/SIAroadmap97b.pdf

[2] G. A. Katopis, "Delta-I noise specification for high-performance computing machine," Proc. IEEE, vol. 73, no. 9, pp. 1405-1415, Sep. 1985

[3] E. Davidson, "Delay factors for mainframe computers," in Proc. Bipolar Circuits Technol. Meeting, 1991, pp. 116-123.

[4] J. M. Jong, V. K. Tripathi, and B. Janko, "Modeling and simulation of switching noise with the associated package resonance for high speed digital circuits," in Proc. Electron. Compon. Technol., 1995, pp. 323-328.

[5] J. M. Wiliamson, M. S. Nakhla, Q.-J. Zhang, and P. Van der Puije, "Ground noise minimization in integrated circuit packages through pin assignment optimization," IEEE Trans. Comp., Packag., Manuf. Technol. B, vol. 19, no. 2, pp. 361-371, May 1996.

[6] Y. Chen, Z. Wu, A. Agrawal, Y. Liu, and J. Fang, "Modeling of delta-I noise in digital electronic packaging," in Proc. IEEE Multi-Chip Module Conf., Santa Cruz, CA, Mar. 15-17, 1994, pp. 126-131.

[7] J. Fan, Y. Ren, J. Chen, D. M. Hockanson, H. Shi, J. L. Drewniak, T H. Hubing, T. P. V. Doren, and E. DuBroff, "RF isolation using power islands in dc power bus design," in IEEE Int. Symp. EMC, 1999, pp. 838-843.

[8] H. J. Liaw and H. Merkelo, "Signal integrity issues at split ground and power planes," in Proc. 46th IEEE Electron. Compon. Technol. Conf., 1996, pp. 752-755.

[9] H. J. Liaw and H. Merkelo, "Crossing the planes at high speed," IEEE Circuit Devices Mag., vol. 13, no. 11, pp. 22-26, Nov. 1997.

[10] C. T. Wu, G. H. Shiue, S. M. Lin, and R. B. Wu, "Composite effects of reflections and ground bounce for signal line through a split power plane," IEEE Trans. Adv. Packag., no. 2, pp. 297-301, May 2002.

[11] C. Schuster and W. Fichtner, "Parasitic modes on printed circuit boards and their effects on EMC and signal integrity," IEEE Trans. Electromagn. Compat., vol. 43, no. 4, pp. 416-425, Nov. 2001.

[12] D. De Zutter, "The FDTD-method for EMC-problems with application to electrostatic discharge and delta-I noise calculations," in IEEE Int. Symp. Electromagn. Compat., 1997, pp. 226-230.

[13] S. Van Den Berghe, F. Olyslager, D. De Zutter, J. De Moerloose, and W Temmerman, "Study of the ground bounce caused by power plane resonance," IEEE Trans. Electromagn. Compat., vol. 40, no. 2, pp. 111-119, May 1998.

[14] H. Shi, J. Fan, J. L. Drewniak, T. H. Hubing, and T. P. Van Doren, "Modeling multilayered PCB power-bus designs using an MPIE based circuit extraction technique," in IEEE Int. Symp. EMC, 1998, pp. 647-651.

[15] A. E. Ruehli and A. C. Cangellaris, "Application of the partial element equivalent circuit (PEEC) method to realistic printed circuit board problem," in IEEE Int. Symp. EMC, 1998, pp. 182-187.

[16] B. Archambeault and A. E. Ruehli, "Analysis of power/ground-plane EMI decoupling performance using the partial-element equivalent circuit technique," IEEE Trans. Electromagn. Compat., vol. 43, no. 4, pp. 437-445, Nov. 2001.

[17] S. W. Leung, L. Wang, and C. M. Ip, "Modeling of the ground bounce effect on PCBs for high-speed digital circuits," in IEEE Int. Symp. EMC, 1999, pp. 110-115.

[18] R. F. Harrington, Time-Harmonic Electromagnetic Field. New York: McGraw-Hill, 1977, p. 127.
Chun-Te Wu was born in Tainan, Taiwan, R.O.C., in 1968. He received the B.S. degree in electrical engineering from Chung Yuan Christian University, Taiwan, in 1990, and the M.S. and Ph.D. degree in electrical engineering from National Taiwan University, Taipei, Taiwan, in 1995 and 2002, respectively.

He is currently an Assistant Professor in the Department of Communication Engineering, Da-Yeh University, Chang-Hua, Taiwan. His areas of interest include computational electromagnetics, thin-slot formalism for finite-difference time domain analysis, and the issues of signal integrity. 
Yi-Hsin Pang was born in Kaohsiung, Taiwan, R.O.C., in 1972. He received the B.S., M.S., and Ph.D. degrees in electrical engineering from National Taiwan University, Taipei, Taiwan, in 1994, 1996, and 2003, respectively.

Since January 2004, he has been with the Industrial Technology Research Center, Hsinchu, Taiwan, as a Design Engineer in the High-Frequency IC Design Department. His areas of interest include computational electromagnetics, antenna analysis and design, and RFIC design.
Ruey-Beei Wu (SM'97) received the B.S.E.E. and Ph.D. degrees from National Taiwan University, Taipei, Taiwan, R.O.C., in 1979 and 1985, respectively.

He then joined the faculty of the Department of Electrical Engineering, National Taiwan University, where he is currently a Professor. He is also with the Graduate Institute of Communications Engineering, which was established in 1997. From March 1986 to February 1987, he was a Visiting Scholar with the IBM Corporation, East Fishkill, NY. From August 1994 to July 1995, he was with the Electrical Engineering Department, University of California at Los Angeles. From May 1998 to April 2000, he was Director of the National Center for High-Performance Computing. From November 2002 to July 2004, he served as Director of the Department of Planning and Evaluation, National Science Council, for the coordination of the national science and technology development. Since August 2004, he has been Chairman of the Department of Electrical Engineering, National Taiwan University. His areas of interest include computational electromagnetics, transmission-line and waveguide discontinuities, microwave and millimeter-wave planar circuits, and interconnection modeling for computer packaging. He has authored or coauthored over 50 publications appearing in IEEE publications and over 70 papers appearing in international conferences.

Dr. Wu is a member of the Phi Tau Phi Scholastic Society, the Chinese Institute of Engineers, the Chinese Institute of Electrical Engineers, and the International Union of Radio Science (URSI). 